\title{
Multiwavelet based Texture Features for Content based Image Retrieval
}

\author{
P.V.N.Reddy \\ Alfa College of Engineering \&Technology, \\ Allagadda, Kurnool, \\ Andhra Pradesh, India.
}

\author{
K.Satya Prasad \\ JNTU College of Engineering, \\ Kakinada, \\ Andhra Pradesh, India.
}

\begin{abstract}
Content based image Retrieval has become one of the most active research areas in the past few years .CBIR system using multiwavelet based features with high retrieval rate and less computational complexity is proposed in this paper. Multiwavelets offer simultaneous orthogonality, symmetry and short support. This property made it a powerful tool for feature extraction of images in the database. Texture features are obtained by computing the energy, standard deviation and mean on each sub band of the multiwavelet decomposed image. To check the retrieval performance texture database of 999 texture images are taken from brotatz album. We have done the comparison of results using mean, energy and standard deviation features and performed that standard deviation gives better results than mean and energy features .Euclidean distance, Canberra distance and Manhattan distance is used as similarity measure in the proposed CBIR system
\end{abstract}

\section{Key words}

CBIR, feature extraction, multiwavelet transform, standard deviation, Euclidean distance \& Canberra distance Manhattan distance.

\section{INTRODUCTION}

With the rapid growth of digital image and video, content Based image retrieval (CBIR) has become important research area to help people to search and retrieve useful information. High retrieval efficiency and less computational complexity are the desired characteristics of CBIR system. CBIR finds applications in advertising, medicine, crime detection, entertainment and digital libraries. Computational Complexity and retrieval efficiency are the key objectives in the design of CBIR system [1]. However, designing of CBIR system with these objectives becomes difficult as the size of image data base increases. CBIR based on color, texture, shape and edge information are available in the literature $[2,3,4$, $5,6]$.This paper describes an image retrieval technique based on multiwavelet texture features. Texture is an important feature of natural images. Features of an image should have a strong relationship with semantic meaning of the image. CBIR system retrieves the relevant images from the image data base for the given query image, by comparing the feature of the query image and images in the database. Relevant images are retrieved according to minimum distance or maximum similarity [7] measure calculated between features of query image and every image in image database.

CBIR systems can be based on many features viz, texture, color, shape and edge information. Texture contains important information about the structural arrangement of surfaces and their relationship to the surroundings. Varieties of techniques are developed for texture analysis $[8,9]$.Most of the texture features are obtained from the application of a local operator, statistical analysis or measurement in transform domain.

Gabor filter (or Gabor wavelet) is widely adapted to extract texture features from the images for image retrieval $[5,7,8,9,10$, and 11$]$ and has been shown to be very efficient. Manjunath and $\mathrm{Ma}$ [5] have shown that image retrieval using Gabor features outperforms that using pyramid-structured wavelet transform [PWT] features, tree structured wavelet transform [TWT] features. In the proposed method an image is represented in the multiwavelet transform domain. Normalized standard deviation \& energy computed on each sub band of the multiwavelet decomposed image is used as feature vector to represent every image in the image database. Wavelet transforms should have the property of orthogonality, symmetry, short support and higher order approximation. Due to implementation constraints scalar wavelets do not satisfy all these properties simultaneously.Multi wavelets $[4,5]$ which are wavelets generated by finite set of scaling functions, have several advantages in comparison to scalar wavelets .One of the advantages is that a multi wavelet can posses the orthogonality and symmetry simultaneously $[9,11,12]$. Thus multiwavelets offer the possibility of superior performance and high degree of freedom for image processing applications, compared with scalar wavelets. Hence, the proposed method based on multiwavelets is giving better performance in terms of computational complexity and retrieval efficiency over Gabor wavelet features based CBIR system. 
This paper is organized as follows:

In section 2, multiwavelet transform and its advantages over wavelet transform are reviewed. Multiwavelet based CBIR system and its algorithm is discussed in section 3. Experimental results are given in section 4.Concluding remarks and feature directions are given in section 5 .

\section{MULTIWAVELET TRANSFORM}

Multiwavelets were defined using several wavelets with several scaling functions [7]. Multiwavelets have several advantages in comparison with scalar wavelet [8]. The features such as compact support, Orthogonality, symmetry, and high order approximation are the base features for this transformation. A scalar wavelet cannot possess all these properties at the same time. On the other hand, a multiwavelet system can simultaneously provide perfect representation while preserving length (Orthogonality), good performance at the boundaries (via linear-phase symmetry), and a high order of approximation (vanishing moments) [10]. Thus multiwavelets offer the possibility of superior performance and high degree of freedom for image processing applications, compared with scalar wavelets. The study of multiwavelets was initiated by Goodman, Lee and Tang. The special case of Multiwavelets with multiplicity 2 and support $(0,2)$, was studied by Chui and Lian. When a multiresolution analysis is generated using multiple scaling functions and wavelet functions, it gives rise to the notion of multiwavelets [5]. A multiwavelet with ' $r$ ' scaling functions and ' $r$ ' wavelet functions is said to have multiplicity ' $r$ '. When $r=1$, with one scaling function and one wavelet function, the multiwavelet system reduces to scalar wavelet system. In Multiwavelet transforms they have two or more scaling functions and wavelet functions. The set of scaling functions are represented using the vector notation

$\phi(t)=\left[\phi_{1}(t) \phi_{2}(t) \ldots \ldots \ldots \phi_{r}(t)\right]^{T}$

Where $\Phi(t)$ is called the multi-scaling function. The multiwavelet function is defined from the set of wavelet function

$\psi(t)=\left[\psi_{1}(t) \psi_{2}(t) \ldots \ldots \ldots . . \psi_{r}(t)\right]^{T}$

When $\mathrm{r}=1, \psi(t)$ is called a scalar wavelet or simply wavelets. Multiwavelets differ from scalar wavelet systems in requiring two or more input streams to the multiwavelet filter bank. Multiwavelets are an extension of the scalar wavelet to the vector case. As in the scalar wavelet case, the theory of multiwavelets is based on the idea of multiresolution analysis (MRA). The difference is that multiwavelets have several scaling functions. The multi scaling function and the multiwavelet function will satisfy matrix dilation equations,

$$
\begin{aligned}
& \phi(t)=\sqrt{2} \sum_{k=-\infty}^{\infty} H_{k} \phi(2 t-k) \\
& \psi(t)=\sqrt{2} \sum_{k=-\infty}^{\infty} G_{k} \phi(2 t-k)
\end{aligned}
$$

The filter coefficients $H_{k}$ and $G_{k}$ are $N$ by $N$ matrices instead of scalar. Corresponding to each multiwavelet system, there is a matrix-valued with multi-rate filter bank. A multiwavelet filter bank has "taps" that are N $\times \mathrm{N}$ matrices. One desirable feature of any transform used in image retrieval is the amount of energy compaction achieved. A filter with good energy compaction properties can decorrelate a fairly uniform input signal into a small number of scaling coefficients containing most of the energy and a large number of sparse wavelet coefficients. Therefore better performance is obtained when the wavelet coefficients have values clustered about zero with little variance. Thus multiwavelets have the potential to offer better representative quality than the conventional scalar transforms. Finally, multiwavelets can achieve better level of performance than scalar wavelets with similar computational complexity. Wavelets are useful tools for signal processing applications such as image retrieval and denoising.

\begin{tabular}{|l|l|}
\hline LL & LH \\
\hline HL & HH \\
\hline
\end{tabular}

\begin{tabular}{|l|l|l|l|}
\hline $\mathrm{L}_{1} \mathrm{~L}_{1}$ & $\mathrm{~L}_{1} \mathrm{~L}_{2}$ & $\mathrm{~L}_{1} \mathrm{H}_{1}$ & $\mathrm{~L}_{1} \mathrm{H}_{2}$ \\
\hline $\mathrm{L}_{2} \mathrm{~L}_{1}$ & $\mathrm{~L}_{2} \mathrm{~L}_{2}$ & $\mathrm{~L}_{2} \mathrm{H}_{1}$ & $\mathrm{~L}_{2} \mathrm{H}_{2}$ \\
\hline $\mathrm{H}_{1} \mathrm{~L}_{1}$ & $\mathrm{H}_{1} \mathrm{~L}_{2}$ & $\mathrm{H}_{1} \mathrm{H}_{1}$ & $\mathrm{H}_{1} \mathrm{H}_{2}$ \\
\hline $\mathrm{H}_{2} \mathrm{~L}_{1}$ & $\mathrm{H}_{2} \mathrm{~L}_{2}$ & $\mathrm{H}_{2} \mathrm{~L}_{1}$ & $\mathrm{H}_{2} \mathrm{~L}_{2}$ \\
\hline
\end{tabular}

Figure.1 Image decomposition after a single level scaling for (a) Scalar wavelets and (b) multiwavelets
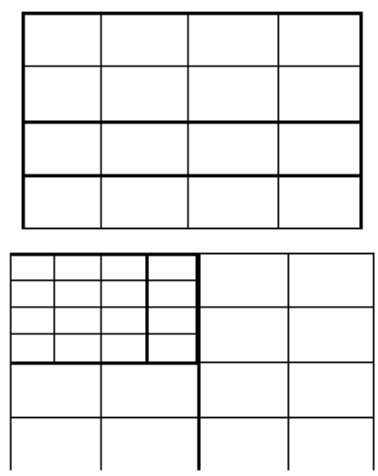

Figure.2 Conventional iteration of multiwavelet decomposition. 
During a single level of decomposition using a scalar wavelet transform, the 2- D image data is replaced by four blocks corresponding to the sub bands representing either low pass or high pass in both dimensions. These sub bands are illustrated in Fig. 1. The multi-wavelets used here have two channels, so there will be two sets of scaling coefficients and two sets of wavelet coefficients. Since multiple iteration over the low pass data is desired, the scaling coefficients for the two channels are stored together. Likewise, the wavelet coefficients for the two channels are also stored together. The multi-wavelet decomposition sub bands are shown in Fig.2. For multi-wavelets the $\mathrm{L}$ and $\mathrm{H}$ have subscripts denoting the channel to which the data corresponds. For example, the sub band labeled $\mathrm{L} 1 \mathrm{H} 2$ corresponds to data from the second channel high pass filter in the horizontal direction and the first channel low pass filter in the vertical direction. This shows how a single level of decomposition is done. In practice, there is more than one decomposition performed on the image. Successive iterations are performed on the low pass coefficients from the pervious stage to further reduce the number of low pass coefficients. Since the low pass coefficients contain most of the original signal energy, this iteration process yields better energy compaction. After a certain number of iterations, the benefits gained in energy compaction becomes rather negligible compared to the extra computational effort. Usually five levels of decomposition are used.

\section{CBIR ARCHITECTURE}

The objective of the proposed work is to study the texture features in image retrieval. The basic architecture of CBIR system is shown in figure 3 . An improved method based on multiwavelet transform for CBIR system is proposed in this work. There are two issues in building a CBIR system.

1. Every image in the image data base is to be represented efficiently by extracting significant features.

2. Relevant images are to be retrieved using similarity measure between query and every image in the image data base.

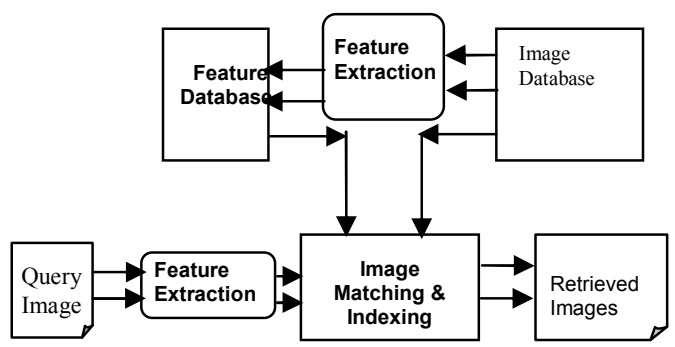

Figure3 CBIR System Architecture

The performance of the proposed CBIR system can be tested by retrieving the desired number of images from the database. The average retrieval rate and retrieval time are the main performance measures in the proposed CBIR system. The average retrieval rate is known as the average percentage number of images belonging to the same images as the query image in the top ' $\mathrm{N}$ ' matches. ' $\mathrm{N}$ ' indicates the number of retrieved images.

\subsection{Proposed Algorithm}

The basic steps involved in the proposed CBIR system includes database processing and resizing, creation and normalization of feature database, comparison and image retrieval. Steps of the proposed algorithm are as follows.

1. Decompose each image in the multi wavelet domain.

2. Compute the standard deviation (SD),energy and mean of the multi wavelet decomposed image.

Standard Deviation is given as

$$
\sigma_{k}=\sqrt{\frac{1}{M x N} \sum_{i=1}^{M} \sum_{j=1}^{N}\left(w_{k}(i, j)-\mu_{k}\right)^{2}}
$$

Where,

$\mathrm{W}_{\mathrm{k}}=$ Co-efficient of $\mathrm{k}^{\text {th }}$ multiwavelet

Decomposed sub-band.

$\mu_{\mathrm{k}} \quad=$ Mean value of $\mathrm{k}^{\text {th }}$ sub-band

$M x N=$ Size of the multiwavelet decomposed sub-band.

The resulting SD vector is

$\bar{f}=\left[\sigma_{1}, \sigma_{2}, \sigma_{3}, \ldots \ldots . \sigma_{n}\right]$

Energy is given as

$$
E_{k}=\frac{1}{M \times N} \sum_{i=1}^{M} \sum_{j=1}^{N}\left|W_{i j}\right|
$$

3. Normalize the SD vector to the range [0 1] for every image in the database.

$$
\bar{f}_{C T}=\frac{\bar{f}-\mu_{\bar{f}}}{\sigma_{\bar{f}}}
$$

Where,

$\mu_{\bar{f}}, \sigma_{\bar{f}}$ are the mean and the standard deviation of $\bar{f}$.

This normalized SD or energy or mean vectors are used to create the feature database.

4. Apply query image and calculate the feature vector as given in steps 2 to 3 .

5. Calculate the similarity measure using Euclidean distance, Canberra distance and Manhattan distance.

6. Retrieve all relevant images to query image based on minimum Euclidean distance or Canberra distance or Manhattan distance. 


\subsection{Retrieval procedure for texture images}

In this section texture image data base used for experimental purpose, feature database creation and image retrieval method are discussed.

Texture image data base

The texture database used in our experiment consists of 999 texture images. For creating this database 111 different texture classes are used from Brodatz texture photographic album.

Feature data base creation

Each image from the database was analyzed using multiwavelet transform. For constructing the feature vector, feature parameters such as energy, standard deviation and mean are computed separately on each subband and are stored in vector form. The assumption of this approach is that the energy distribution in the frequency domain identifies a texture. The energy and standard deviation of decomposed subbands are computed as follows:

Energy $E_{k}=\frac{1}{M \times N} \sum_{i=1}^{M} \sum_{j=1}^{N}\left|W_{i j}\right|$

Standard deviation

$\sigma_{k}=\left[\frac{1}{M \times N} \sum_{i=1}^{N} \sum_{j=1}^{M}\left(W_{i j}-\mu_{i j}\right)^{2}\right]^{\frac{1}{2}}$

Where $W(i, j)$ is the multiwavelet- decomposed subband, $M \times N$ is the size of multiwaveletdecomposed subband, $\mathrm{k}$ is the number of subbands and $\mu_{i j}$ is the subband mean value.

A feature vector is now constructed using $E_{k}, \mu_{i j}$ , and $\sigma_{k}$ as feature components. Length of feature vector will be equal to (No. of subbands $\times$ No. of feature parameters used in combination) elements.

Resulting feature vectors as follows:

Using only energy feature measures

$\bar{f}_{E}=\left[\begin{array}{ll}E_{1} E_{2} \ldots \ldots \ldots . . & E_{K}\end{array}\right]$

Using only standard deviation feature measure

$\bar{f}_{\sigma}=\left[\sigma_{1} \sigma_{2} \ldots \ldots \ldots \sigma_{k}\right]$

For creation of feature database above procedure is repeated for all the image and these feature vectors stored in feature database.

\subsection{Image retrieval method}

A query image is any one of the images from image database. This query image is processed to compute the feature vector as in section 3.2. Traditional distance metric, Canberra distance and Manhattan metrics are used to compute the similarity or match value for given pair of images.
If $\mathrm{x}$ and $\mathrm{y}$ is two-dimensional feature vectors of database image and query image respectively, then these distance metrics are defined as:

The Euclidean or L2 metric is

$d_{E}(x, y)=\sqrt{\sum_{i=1}^{d}\left(x_{i}-y_{j}\right)^{2}}$

Euclidean distance is not always the best metric. The fact that the distances in each dimension are squared before summation, places great emphasis on those features for which the dissimilarity is large. Hence it is necessary to normalize the individual feature components before finding the distance between two images. This has been taken care of in Canberra distance metric, which motivates us to use Canberra distance metric as dissimilarity measure. This is also supported by our experimental results given Table 1 . Canberra distance is given by

$\operatorname{Canb}(x, y)=\sum_{i=1}^{d} \frac{\left|x_{i}-y_{i}\right|}{\left|x_{i}\right|+\left|y_{i}\right|}$

In above Eq. the numerator signifies the difference and denominator normalizes the difference. Thus distance values will never exceed one, being equal to one whenever either of the attributes is zero. Thus it would seem to be a good expression to use, which avoids scaling effect. It is obvious that the distance of an image from itself is zero. The distances are stored in increasing order and the closest sets of patterns are retrieved.

The Manhattan distance is given by

$D_{q i}^{M} \sum_{i=1}^{n}\left|\bar{f}_{q j}-\bar{f}_{i j}\right|$

The performance is measured in terms of the average retrieval rate, which is defined as the average percentage number of patterns belonging to the same image as the query pattern in the top 16 matches.

\section{Experimental Results}

Table1 provides a detailed comparison of average retrieval accuracy for entire database and retrieval time for query image using multiwavelet with feature parameters such as mean, energy and standard deviation. Comparison of retrieval performance using Euclidean distance, Canberra distance and Manhattan

distance metric is also presented. The proposed system has been implemented using MATLAB7.2 on Pentium-4, 3.00 GHz machine

Retrieved top 16 similar images from the database using multiwavelet for a sample Query Image as shown in figure 4. 


\section{Query Image}
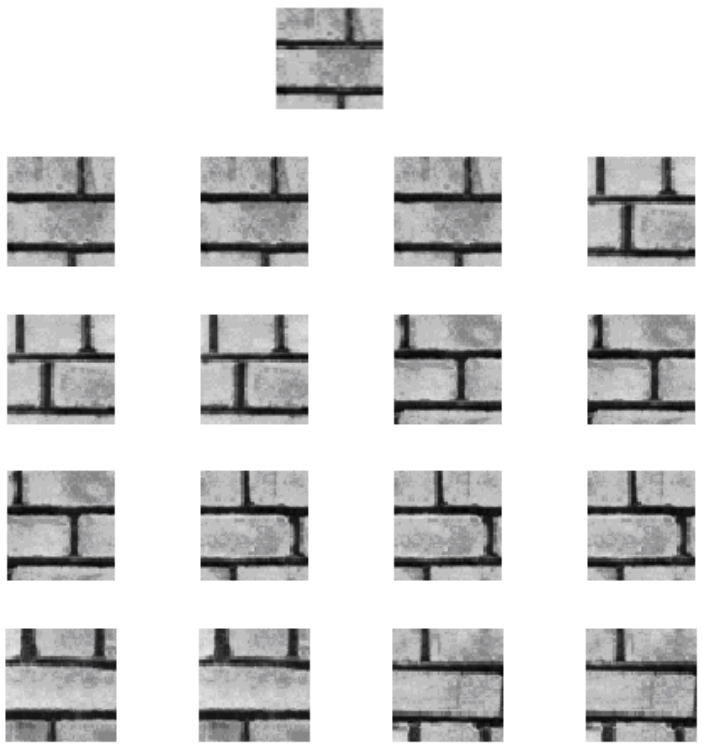

Figure 4.Retrieved top 16 images for a sample query of 311 in the data base

Figure 5. Shows retrieval performance of multiwavelet transform according to the number of top matches considered. From that figure it is clear that the retrieval performance using standard deviation is superior to energy and mean features.

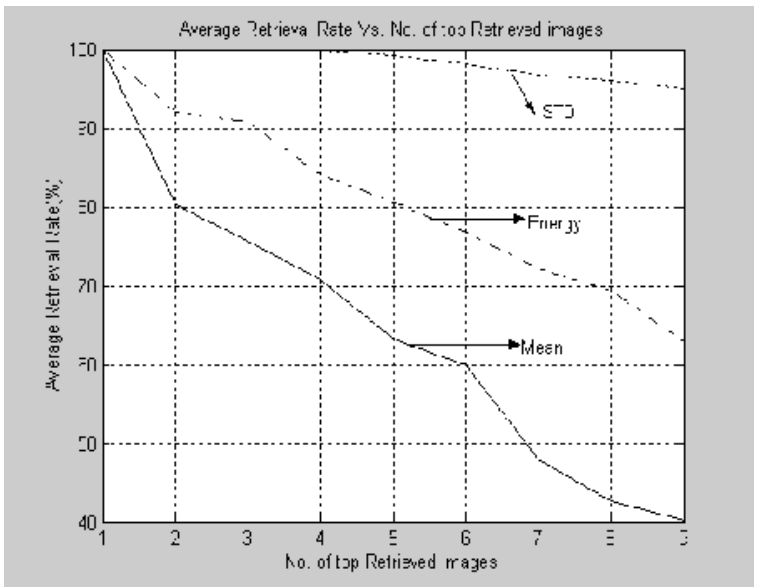

Figure 5.comparative average retrieval rate

Table 1: Average retrieval rate of all the database images

\begin{tabular}{|l|c|c|c|c|c|c|c|c|c|}
\hline & \multicolumn{7}{|c|}{ Number of top matches } \\
\hline Features & 1 & 2 & 3 & 4 & 5 & 6 & 7 & 8 & 9 \\
\hline Std & 100 & 100 & 100 & 100 & 99.28 & 98.12 & 96.73 & 96.1 & 95.02 \\
\hline Energy & 100 & 92.02 & 90.73 & 84.12 & 80.56 & 76.73 & 72.14 & 69.36 & 62.93 \\
\hline Mean & 100 & 80.22 & 75.69 & 70.78 & 63.24 & 59.91 & 47.95 & 42.76 & 40.31 \\
\hline
\end{tabular}

\section{CONCLUSION}

Content based image retrieval using multiwavelet based texture features is proposed. A detailed comparison with the performance of features such as mean, standard deviation and energy using Euclidean distance Manhattan distance and Canberra distance function is presented .Among all these feature parameters we found that standard deviation with Euclidean distance gives best retrieval results while mean feature alone performs poor.

\section{REFERENCES}

[1] Arnold. W. M. Smeulders, M. Worring, S. satini,A. Gupta, R. Jain. Content - Based ImageRetrieval at the end of the Early Years. IEEE Transactions on Pattern analysis and Machine Intelligence, Vol. 22, No. 12, pp 13491380,2000 .

[2] Belongie S., Carson C., et al., Color - and Texture Based Image Segmentation using EM and its Application to Content-Based Image Retrieval. Proceedings of 8th International Conference on Computer Vision, 1998. 
[3] Manesh Kokare, B .N.Chatterji and P.K.Biswas. A Survey on current content based Image Retrieval Methods, IETE Journal of Research, Vol. 48, No. $3 \& 4$, pp 261-271, 2002.

[4] Gupta. A.Visual Information Retrieval Technology: A Virage Perspective, Virage Image Engine. API Specification, 1997.

[5] Smith. J and Chang S.F. Visual SEEK: a fully automated content-based image query system. Proceedings of ACM Multimedia 96, pp 87-98, 1996.

[6] N. Monserrat, E. de Ves, P. Zuccarello. Proceedings of GVIP 05 Conference, December 2005.

[7] S. Satini, R. Jain. Similarity Measures. IEEE Transactions on pattern analysis and machine Intelligence, Vol.21, No.9, pp 871-883, September 1999.

[8] Hiremath.P.S, Shivashankar . S. Wavelet based features for texture classification, GVIP Journal, Vol.6, Issue 3, pp 55-58, December 2006.

[9] B. S. Manjunath and W.Y. Ma. Texture Feature for Browsing and Retrieval of Image Data. IEEE Transactions on Pattern Analysis and Machine Intelligence, Vol. 8, No. 8, 1996.

[10] X. Wan and C. C. J. Kuo. Color distribution analysis and quantization for image retrieval. In SPIE proceedings, vol.2670, February 1996.

[11] Sclaroff. S., Taycher. L., La Cascia. M. Image Rover: a content-based browser for the world wide web. In: IEEE Workshop on Content Based Access of Image and Video Libraries, pp 2-6, 1997.

[12] Jain.A.K,Vailaya.A. Image retrieval using color and shape. Pattern Recognition 29 (8), pp 12331244, 1996.

[13] Cohen. S. D., Guibas .L.J. Shape-based image retrieval using geometric hashing. In: Proceedings of ARPA Image understanding Workshop, pp 669-674, 1997.

[14] Arti Khaparde, M.Maghavilatha, 2006, Iris Recognition using Gabor filters and xeta square statistics, Proceeding of IFToMM-PCEA International conference PICA-July 2006, Nagpur, India.

[15] Arti haparde B.L.Deekshatulu, M.Madhavilatha, 2008, Content Based Image Retrieval Using Independent component Analysis, International Journal of Computer Science and Netwrok Security, 8(4), pp. 327-332.

[16] A.J.Bell and T.J.Sejnowski, 1997, the 'independent components' of natural scenes are edge filters. Vision Research, 37, pp. 3327-3338.
[17] David A Clause, M.ED Jerni,GAN, 2000 Designing Gabor filters for optimal texture separability, Pattern Recognition, 33, pp.18351849.

[18] Dengsheng Zhang, Aylwin wong, Maria Indrawan, and Guojun $\mathrm{Lu}, 2003$, Content Based Image Retrieval using Gabor Texture features, available online, Australia.

[19] Manthalakar R, .Biswas P.K, Chatterji B. N, 2003, Rotation and scale invariant texture features using discrete wavelet packed transform. Pattern Recognition letter 24(14), pp. 24552462.

[20] Mallat S, "A Wavelet Tour of Signal Processing". New York: Academic, 1998.

[21] Vasily Strela, Peter Niels Heller, Gilbert Strang, Pankaj Topiwala, and Christopher Heil, "The Application of Multiwavelet Filter banks to Image Processing", IEEE Transactions on image processing, vol. 8, no.4, April 1999. Pp.548-563.

[22] Strang.G and T. Nguyen, "Wavelets and Filter Banks". Wellesley, MA:Wellesley-Cambridge Press, 1995.

[23] Wonkookim and Ching Chung, "On preconditioning multiwavelet system for image compression", International Journal of Wavelets, Multiresolution and Information Processing, Vol. 1, No. 1 (2003), pp.51-74.

[24] Michael B. Martin and Amy E. Bell , "New Image Compression Techniques using Multiwavelets and Multiwavelet Packets", IEEE Transactions on image processing, vol. 10, No. 4, , April 2001.

\section{ABOUT THE AUTHORS}

P.V.N. Reddy is currently working as HOD of Electronics \& Communications Engineering, Alfa College of Engineering \& Technology, Allagadda, Kurnool, Andhra Pradesh, India.

Dr. K. Satya Prasad is currently working as Rector, JNTUK Kakinada \& Professor of Electronics \& Communications Engineering. He has more than 28 years of Experience in teaching and 20 years of R \& D. He is an expert in Digital Signal Processing. He has produced $4 \mathrm{PhD}$ 's and guiding $10 \mathrm{PhD}$ scholars. $\mathrm{He}$ has published more than 30 technical papers in national and International Journals and conferences. 